\title{
The fusion process of interval opinions based on the dynamic bounded confidence
}

Haiming Liang ${ }^{\mathrm{a}}$, Cong-Cong Li ${ }^{\mathrm{a}}$, Yucheng Dong ${ }^{\mathrm{a}^{*}}$, Yanping Jiang ${ }^{\mathrm{b}}$

a. Business School, Sichuan University, Chengdu 610065, China

b. School of Business administration, Northeastern University, Shenyang 110004, China

\begin{abstract}
In this paper, we propose a novel opinion dynamics model that is based on bounded confidence and termed interval opinion dynamics with the dynamic bounded confidence. In this opinion dynamics model, the agents express their opinions in numerical intervals, and the bounded confidences vary in a specified interval as time varies (i.e., dynamic bounded confidence). Based on several theoretical analyses of the proposed opinion dynamics, we propose conditions that are sufficient to form a consensus or fragmentations among the agents. Moreover, we also design several simulation experiments to investigate the effects of the dynamic bounded confidence and interval widths on the proposed opinion dynamics and to illustrate the differences between the proposed model and the original opinion dynamics with bounded confidence.
\end{abstract}

Keywords: Opinion dynamics, Fusion process, Interval opinions, Dynamic bounded confidences, Consensus

\section{Introduction}

Opinion dynamics is a fusion process of individual opinions that can be defined as a group of interacting agents who continuously update their opinions regarding the same issue based on the established fusion rules and reach a consensus (or fragmentation) in the final stage.

In opinion dynamics, the establishment of the fusion rule is the core problem [1]. Recently, some research results regarding the fusion rule have been reported, such as the voter model [2], the persuasiveness and supportiveness model [3], the bounded confidence model [4,5] and the Alexford model [6]. Among these existing models, the use of the bounded confidence model as the fusion rule has become a topic of intensive research in recent years.

The bounded confidence model assumes that the agents only communicate with the peers

* Corresponding author. Tel.: +86 2882673492.

E-mail address: ycdong@mail.xjtu.edu.cn (Y. Dong). 
who hold similar opinions and tend to ignore the peers with sufficiently different opinions [7]. The earliest bounded confidence models were introduced independently by Hegselmann and Krause (HK model) [4] and Deffuant and Weisbuch (DW model) [5]. In the HK model, the agents synchronously update their opinions by averaging all of the opinions in their confidence set [4], and in the DW model, the agents adhere to pairwise-sequential updating mechanisms [5]. Based on the original HK and DW models, the following different types of bounded confidence models have been proposed: (i) The agent-based homogeneous models [8-13], (ii) The agent- based heterogeneous models [14-16], (iii) The density-based homogeneous models [17-20], and (iv) The density-based heterogeneous models [9, 15, 21].

Previous studies have significantly advanced the bounded confidence model. In this study, we propose the interval opinion dynamics with dynamic bounded confidence model. The study is motivated by the following two aspects:

(1) In the existing studies, each agent uses a crisp number to express his/her opinion, i.e., a crisp opinion. However, in the practical processes of opinion dynamics, the opinions of the agents often exhibit uncertainty. Thus, it is necessary to investigate the effects of the uncertain opinions on opinion dynamics. Generally, interval opinions are the most basic formats of uncertain opinions [22-24]. Thus, in the present study, the fusion of interval opinions will provide a foundation for investigating the effects of uncertain opinions on opinion dynamics.

(2) Bounded confidence is the basic assumption of the opinion dynamics problem. In the existing studies, there are two types of bounded confidences, i.e., homogeneous and heterogeneous bounded confidences. The former refers to cases in which all of the agents have a uniform bounded confidence. The latter refers to cases in which each agent has his/her own bounded confidence. However, in the practical processes of opinion dynamics, the bounded confidence will vary dynamically. For example, with deep interactions, the trust between the agents may be strengthened. In such situations, their bounded confidences will increase over time, whereas with increases in conflicts of opinions, the agents' bounded confidences decrease over time. Therefore, it is necessary to study the opinion dynamics with the assumption of dynamic bounded confidence.

The aim of this paper is to discuss the fusion process of interval opinions with dynamic bounded confidence. The remainder of this paper is arranged as follows. In Section 2, we introduce the HK model. Next, in Section 3, we propose the interval opinion dynamics model with dynamic bounded confidence. In section 4 , we conduct the theoretical analyses of the proposed model. In the theoretical analyses, we provide the conditions that are sufficient for the formation of a consensus or fragmentations among the agents. Furthermore, in Section 5 
we design some simulation experiments to investigate the effects of the dynamic bounded confidence and the interval widths on the proposed opinion dynamics and illustrate the differences between the proposed model and the original HK model. Finally, concluding remarks are presented in Section 6.

\section{Preliminary: the HK model}

In this section, we introduce the original HK model, which will provide a foundation for this study.

The fundamental difference between the DW and HK models is the number of agents that communicate, which is labelled as the communication regime. In this paper, without loss of the generality, we adopt the original HK model as the foundation model. We could propose a similar study that involved the adoption of the original DW model.

The HK model [4] is briefly introduced as follows:

Consider a set of agents, $A=\left\{A_{1}, A_{2}, \ldots, A_{N}\right\}$ and a discrete time $t, t=0,1,2, \ldots$. The crisp opinion of an agent $A_{i} \in A$ at time $t$ is represented by $x_{i}(t) \in[0,1]$. Let $X(t)=\left(x_{1}(t)\right.$, $\left.x_{2}(t), \ldots, x_{N}(t)\right)^{T}$ be the vector of the opinions of all of the agents at time $t$ that is called the opinion profile. Let $\varepsilon_{i}$ be the bounded confidence of agent $A_{i}$. Agent $A_{i}$ only considers the opinions that differ from his/her own opinion by not more than $\varepsilon_{i}$. When $\varepsilon_{i}=\varepsilon_{j}$ for $i, j=1,2, \ldots, N$, the model is termed the HK model with the homogeneous bounded confidence; otherwise, the model is termed the HK model with the heterogeneous bounded confidence.

The process of the HK model includes three steps:

(1) Determinations of the confidence sets.

Let $I\left(A_{i}, x_{i}(t)\right)$ be the confidence set of agent $A_{i}$ at time $t$, and $I\left(A_{i}, x_{i}(t)\right)$ is determined as:

$$
I\left(A_{i}, x_{i}(t)\right)=\left\{A_{j}|| x_{i}(t)-x_{j}(t) \mid \leq \varepsilon_{i}, j=1,2, \ldots, N\right\}, \quad i=1,2, \ldots, N .
$$

(2) Calculations of the weights.

Let $w_{i j}(t)$ be the weight that agent $A_{i}$ assigns to agent $A_{j}$ at time $t$. Using the obtained set $I\left(A_{i}, x_{i}(t)\right)$, we can calculate the weight $w_{i j}(t)$ as:

$$
w_{i j}(t)=\left\{\begin{array}{ll}
0, & A_{j} \notin I\left(A_{i}, x_{i}(t)\right) \\
1 /\left|I\left(A_{i}, x_{i}(t)\right)\right|, & A_{j} \in I\left(A_{i}, x_{i}(t)\right)
\end{array}, \quad i=1,2 \ldots N,\right.
$$

where 14 denotes the cardinality of $I\left(A_{i}, x_{i}(t)\right)$. Clearly, $w_{i j}(t) \geq 0$ and $\sum_{j=1}^{N} w_{i j}(t)=1$. 
(3) Evolutions of the opinions.

The evolutions of the opinions in the HK model are modelled as the weighted arithmetic means of the opinions in the confidence sets, i.e.,

$$
x_{i}(t+1)=w_{i 1}(t) x_{1}(t)+w_{i 2}(t) x_{2}(t)+\cdots+w_{i N}(t) x_{N}(t), \quad i=1,2, \ldots, N .
$$

\section{The proposed model}

In this section, we propose the interval opinion dynamics model with the dynamic bounded confidence. In our proposal, agent $A_{i} \in A$ expresses his/her opinion by a numerical interval $x_{i}(t)=\left[x_{i}^{L}(t), x_{i}^{U}(t)\right]$, where $x_{i}(t) \subseteq[0,1]$. Let $X^{L}(t)=\left(x_{1}^{L}(t), x_{2}^{L}(t), \ldots, x_{N}^{L}(t)\right)^{T}$ and $X^{U}(t)=\left(x_{1}^{U}(t), x_{2}^{U}(t), \ldots, x_{N}^{U}(t)\right)^{T}$ be the lower and upper bounds of the opinion profile, respectively. Let $\varepsilon_{i}(t)$ be the dynamic bounded confidence of agent $A_{i}$ at time $t$, where $0 \leq \varepsilon_{i}(t) \leq \alpha$, and $\alpha$ is the maximum threshold of the dynamic bounded confidence.

Inspired by the HK model, we define the new confidence set $\tilde{I}\left(A_{i}, x_{i}(t)\right)$ as:

$$
\tilde{I}\left(A_{i}, x_{i}(t)\right)=\left\{A_{j} \mid d\left(x_{i}(t), x_{j}(t)\right) \leq \varepsilon_{i}(t), j=1,2, \ldots, N\right\}, \quad i=1,2 \ldots N,
$$

where $d\left(x_{i}(t), x_{j}(t)\right)$ denotes the distance between the interval opinions $x_{i}(t)$ and $x_{j}(t)$.

The distance $d\left(x_{i}(t), x_{j}(t)\right)$ can be calculated using various distance measures (e.g., Manhattan or Euclidean).

When adopting the Manhattan distance measure, the distance $d\left(x_{i}(t), x_{j}(t)\right)$ is given by

$$
d\left(x_{i}(t), x_{j}(t)\right) \frac{\left|x_{i}^{L}(t)-x_{j}^{L}(t)\right|+\left|x_{i}^{U}(t)-x_{j}^{U}(t)\right|}{2} .
$$

When adopting the Euclidean distance measure, the distance $d\left(x_{i}(t), x_{j}(t)\right)$ is given by

$$
d\left(x_{i}(t), x_{j}(t)\right)=\sqrt{\frac{1}{2}\left[\left(x_{i}^{L}(t)-x_{j}^{L}(t)\right)^{2}+\left(x_{i}^{U}(t)-x_{j}^{U}(t)\right)^{2}\right]} .
$$

Regardless of which of the above distance measures we use, similar results are obtained. Without loss of generality, we adopt the Euclidean distance measure in the present study.

The evolutions of the interval opinions in the proposed model are modelled as the weighted arithmetic means of the upper and lower bounds of the interval opinions in the confidence sets, i.e.,

$$
x_{i}^{L}(t+1)=w_{i 1}(t) x_{1}^{L}(t)+w_{i 2}(t) x_{2}^{L}(t)+\ldots+w_{i N}(t) x_{N}^{L}(t), \quad i=1,2, \ldots, N,
$$

and 


$$
x_{i}^{U}(t+1)=w_{i 1}(t) x_{1}^{U}(t)+w_{i 2}(t) x_{2}^{U}(t)+\ldots+w_{i N}(t) x_{N}^{U}(t), \quad i=1,2, \ldots, N,
$$

respectively, where the weight $w_{i j}(t)$ is given by

$$
w_{i j}(t)=\left\{\begin{array}{ll}
0, & A_{j} \notin \tilde{I}\left(A_{i}, x_{i}(t)\right) \\
1 /\left|\tilde{I}\left(A_{i}, x_{i}(t)\right)\right|, & A_{j} \in \tilde{I}\left(A_{i}, x_{i}(t)\right)
\end{array}, \quad i=1,2, \ldots, N .\right.
$$

Let $W(t)=\left(w_{i j}(t)\right)_{N \times N}$. Then, based on Eqs. (5) and (6), the lower and upper bounds of opinion profile are given by

$$
\begin{aligned}
& x^{L}(t+1)=W(t) x^{L}(t) . \\
& x^{U}(t+1)=W(t) x^{U}(t) .
\end{aligned}
$$

The opinion profiles are further determined as

$$
\begin{gathered}
x^{L}(t+1)=W(t) W(t-1) \cdots W(0) x^{L}(0) . \\
x^{U}(t+1)=W(t) W(t-1) \cdots W(0) x^{U}(0) .
\end{gathered}
$$

The proposed model shares a strong linkage to the HK model. If $\varepsilon_{i}(t)=\varepsilon_{i}(t+1)$ and $\varepsilon_{i}(t)=\varepsilon_{j}(t)$ are satisfied for $i, j=1,2, \ldots, N$ and $t=0,1, \ldots$, then the proposed model is reduced to the HK model with the homogeneous bounded confidence. If only $\varepsilon_{i}(t)=\varepsilon_{i}(t+1)$ is satisfied for $i=1,2, \ldots, N$ and $t=0,1, \ldots$, then the proposed model is reduced to the $\mathrm{HK}$ model with the heterogeneous bounded confidence.

\section{Theoretical analysis}

In this section, we propose some theorems on the proposed model to investigate the conditions to form the stabilized result (i.e., consensus and fragmentations).

Before proposing these theorems, we introduce several definitions regarding the consensus, fragmentation, confidence chain, and accumulated weight matrix.

Definition 1: Let $x_{i}(t)=\left[x_{i}^{L}(t), x_{i}^{U}(t)\right]$ and $x_{j}(t)=\left[x_{j}^{L}(t), x_{j}^{U}(t)\right]$ be as before. If $x_{i}(t)$ $=x_{j}(t)$ (i.e., $x_{i}^{L}(t)=x_{j}^{L}(t)$ and $\left.x_{i}^{U}(t)=x_{j}^{U}(t)\right)$, then we call that a consensus among the agents is reached at time $t$; otherwise, if $x_{i}(t) \neq x_{j}(t)$ and $d\left(x_{i}(t), x_{j}(t)\right)>\alpha$, then we call that fragmentations among the agents are formed.

Similar to the confidence chain presented in [25], we define the confidence chain for the proposed model in Definition 2.

Definition 2: Let $s$ and $t(s<t)$ be any two times, and let $A_{i_{0}}, A_{i_{1}}, \ldots, A_{i_{t-s}}$ be a sequence of the $t-s+1$ agents. If $A_{i_{1}} \in \tilde{I}\left(A_{i_{l-1}}, x_{i}(t-l)\right)$ for $l=1,2, \ldots, t-s$, then we call that 
there exists a confidence chain from $A_{i}$ to $A_{k}$ between the times $s$ and $t$.

Similar to the accumulated weight presented in [4], we define the accumulated weight matrix for the proposed model in Definition 3.

Definition 3: Let $s$ and $t(s<t)$ be any two times, and $B(t, s)=\left(b_{i j}(t, s)\right)_{N \times N}$ be the accumulated weight matrix between times $s$ and $t$ where $B(t, s)=W(t-1) \cdots W(s)$.

Clearly, the accumulated weight matrix $B(t, s)$ is a nonnegative row-stochastic matrix, i.e., $b_{i j}(t, s) \geq 0$ and $\sum_{j=1}^{N} b_{i j}(t, s)=1$ for $i, j=1,2, \ldots, N$.

Let $z_{v}=\left[z_{v}^{L}, z_{v}^{U}\right](v=1,2, \ldots, p)$ be the set of numerical intervals, and let $C\left(z_{1}, z_{2}, \ldots, z_{p}\right)$ be the set of all of the convex combinations of these numerical intervals where $C\left(z_{1}, z_{2}, \ldots, z_{p}\right)=\left\{\sum_{v=1}^{p} \eta_{v} z_{v} \mid \eta_{v} \geq 0\right.$ and $\left.\sum_{v=1}^{p} \eta_{v}=1\right\}$. Let $F\left(z_{1}, z_{2}, \ldots, z_{p}\right)=\max _{u, r \in C\left(z_{1}, z_{2}, \ldots, z_{p}\right)} d(u, r)$.

Lemma 1: Let $x_{1}(t), x_{2}(t), \ldots, x_{N}(t)$ be as before. Let $\underline{x}^{L}(t)=\min \left\{x_{i}^{L}(t) \mid i=1,2, \ldots, N\right\}$, $\underline{x}^{U}(t)=\min \left\{x_{i}^{U}(t) \mid i=1,2, \ldots, N\right\}, \quad \bar{x}^{L}(t)=\max \left\{x_{i}^{L}(t) \mid i=1,2, \ldots, N\right\} \quad$ and $\quad \bar{x}^{U}(t)=\max \left\{x_{i}^{U}(t) \mid i=1\right.$, $2, \ldots, N\}$. Then,

$$
F\left(x_{1}(t), \ldots, x_{N}(t)\right) \leq\left(1-\min _{1 \leq i, j \leq N} \sum_{k=1}^{N}\left\{w_{i k}(t), w_{j k}(t)\right\}\right) d(\underline{x}(t), \bar{x}(t))
$$

The proof of Lemma 1 is provided in the Appendix.

Theorem 1: A consensus will be reached if one of the following three conditions is satisfied:

(1) Let $t_{0}, t_{1}, t_{2}, \cdots$ be a sequence of times and $\delta_{1}, \delta_{2}, \cdots$ be a sequence of positive numbers where $0=t_{0}<t_{1}<t_{2}<\cdots, \delta_{1}, \delta_{2}, \cdots \in[0,1]$ and $\lim _{r \rightarrow \infty} \sum_{m=1}^{r} \delta_{m}=\infty$. Then,

$$
\sum_{k=1}^{N} \min \left\{b_{i k}\left(t_{m}, t_{m-1}\right), b_{j k}\left(t_{m}, t_{m-1}\right)\right\} \geq \delta_{m} \text { for } m=1,2, \ldots, \infty \text { and } i, j=1,2, \ldots, n
$$

(2) Let $A_{i}$ and $A_{j}$ be as before, i.e., $i, j=1,2, \ldots, n$. Then the two confidence chains from $A_{i}$ to $A_{k}$ and $A_{j}$ to $A_{k}$ between the times $(m-1) h$ and $m h$ exist because $A_{k} \in A$, $m=1,2, \ldots$ and $h \geq 1$;

(3) Let $\Delta \varepsilon_{i}(t)=\varepsilon_{i}(t+1)-\varepsilon_{i}(t)$. Then, $\Delta \varepsilon_{i}(t) \geq \max _{j=1,2, \ldots, N} d\left(x_{i}(t), x_{j}(t)\right)-\varepsilon_{i}(t)$, for $i=1,2, \ldots, N$.

The proof of Theorem 1 is provided in the Appendix.

Theorem 1 provides some conditions that are sufficient to reach a consensus. We show that a consensus will be reached in the following conditions: the sum of the minimum 
accumulated weights between any two agents is a positive number, and the confidence chain among any two agents exists in the same time interval or the new bounded confidence of each agent is larger than the maximum distance between the current interval opinions.

Lemma 2: Let $B\left(t_{0}, t_{1}\right)$ be the accumulated weight matrix between the times $t_{0}$ and $t_{1}$ $\left(t_{0}<t_{1}\right)$, and let $\mu\left(B\left(t_{0}, t_{1}\right)\right)$ be the smallest positive entry of $B\left(t_{0}, t_{1}\right)$. Then $\mu\left(B\left(t_{0}, t_{1}\right)\right) \geq$ $(1 / N)^{N^{2}-N+2}$ given that $\varepsilon_{i}(t)=\varepsilon_{j}(t)$ for $i, j=1,2, \ldots, n$ and $t=0,1,2, \ldots$

The proof of Lemma 2 is provided in the Appendix.

Theorem 2: Let $L\left(x_{\max }^{(0)}\right)=\max \left\{x_{i}^{U}(0)-x_{i}^{L}(0), i=1,2, \ldots, n\right\}$ and $L\left(x_{\min }^{(0)}\right)=\min \left\{x_{i}^{U}(0)-x_{i}^{L}(0)\right.$, $i=1,2, \ldots, n\}$. If the proposed model reaches a consensus at time $t$, denoted as $x^{c}=\left[x^{c L}, x^{c U}\right]$, then the upper bound of the width of $L\left(x^{c}\right)$ is given as follows:

(1) If $t<N^{2}-N+2$, then

$$
L\left(x^{c}\right) \leq\left[1-(N-1)(1 / N)^{t}\right] L\left(x_{\max }^{(0)}\right)+(N-1)(1 / N)^{t} L\left(x_{\min }^{(0)}\right) .
$$

(2) If $t \geq N^{2}-N+2$ and $\varepsilon_{1}(t)=\varepsilon_{2}(t)=\cdots=\varepsilon_{N}(t)$, then

$$
L\left(x^{c}\right) \leq\left[1-(N-1)(1 / N)^{N^{2}-N+2}\right] L\left(x_{\max }^{(0)}\right)+(1 / N)^{N^{2}-N+2} L\left(x_{\min }^{(0)}\right) .
$$

(3) If $t \geq N^{2}-N+2$ and $\varepsilon_{i}(t) \neq \varepsilon_{k}(t)$ for $\exists i, k \in\{1,2, \ldots, N\}$, then

$$
L\left(x^{c}\right) \leq\left[1-(N-1)(1 / N)^{t}\right] L\left(x_{\max }^{(0)}\right)+(N-1)(1 / N)^{t} L\left(x_{\min }^{(0)}\right) .
$$

The proof of Theorem 2 is provided in the Appendix.

Theorem 2 provides the upper bounds of the widths of the consensus interval opinion.

Theorem 3: Let $\alpha$ be defined as before. We assume there exists $f(t)$ interval opinions $\bar{x}^{1}(t), \bar{x}^{2}(t), \ldots, \bar{x}^{f(t)}(t)$ at time $t, f(t) \geq 2$. Fragmentations are formed if one of the following conditions is satisfied:

(1) Let $\varepsilon_{i}(t)$ and $\varepsilon_{i}(\tilde{t})$ be the dynamic bounded confidence at times $t$ and $\tilde{t}(\tilde{t} \geq t)$, respectively. Then $\varepsilon_{i}(\tilde{t}) \leq \varepsilon_{i}(t)$, for $i=1,2, \ldots, N$.

(2) $d\left(\bar{x}^{g}(t), \bar{x}^{h}(t)\right)>\alpha$, for $g, h=1,2, \ldots, f(t)$.

The proof of Theorem 3 is provided in the Appendix.

Theorem 3 provides the conditions that are sufficient to form fragmentations. We have shown that fragmentations will be formed if the dynamic bounded confidence is decreasing or the distance between any two interval opinions is larger than $\alpha$.

\section{Simulation analysis}

In this section, we propose the simulations to explore the following three issues: the 
effects of the dynamic bounded confidence on the opinion dynamics, the effects of the widths of the interval opinions on the opinion dynamics, and the comparisons between our proposal and the original HK model.

\subsection{The effects of dynamic bounded confidence}

We investigated the effects of the dynamic bounded confidence from two aspects: the stabilized results (consensus or fragmentation), and the consensus time.

Let $S R$ be the flag of the stabilized result, where $S R=1$ denotes a consensus, and $S R=0$ denotes fragmentations. Let $t^{*}$ be the time at which a consensus is reached, i.e., the consensus time. Then, we present Simulation method I to determine the $S R$ and $t^{*}$ values.

\section{Simulation method I:}

Input: $N$ and $\alpha$.

Output: $S R$ and $t^{*}$.

Step 1: Let $t=0$. Interval opinions $x_{i}(t)=\left[x_{i}^{L}(t), x_{i}^{U}(t)\right] \quad(i=1,2, \ldots, N)$ are uniformly and randomly selected from $[0,1]$.

Step 2: Dynamic bounded confidences $\varepsilon_{i}(t) \quad(i=1,2, \ldots, N)$ are uniformly and randomly selected from $[0, \alpha]$.

Step 3: Using Eqs. (7) and (8) obtains the interval opinion $x_{i}(t+1)$ at time $t+1$. If the conditions regarding stabilized results (see Definition 1) are satisfied, then go to Step 4; otherwise, let $t=t+1$, then go to Step 2 .

Step 4: If $x_{i}(t+1)=x_{j}(t+1)$ for $i, j=1,2, \ldots, N$, then $S R=1$ and $t^{*}=t+1$; otherwise, $S R=0$.

Step 5: Output $S R$ and $t^{*}$.

Note 1 On average, the increases of the $\alpha$ values often cause the increases of the dynamic bounded confidences. So, on average, through changing the $\alpha$ values, the effects of dynamic bounded confidences on opinion dynamics can be discussed.

Let $N=1000$. We set different $\alpha$ values, and run the Simulation method I 1000 times, obtaining the consensus ratio values, which reflect the ratio of reaching a consensus in the proposed model. Fig. 1 shows the consensus ratio values for different $\alpha$ values. 


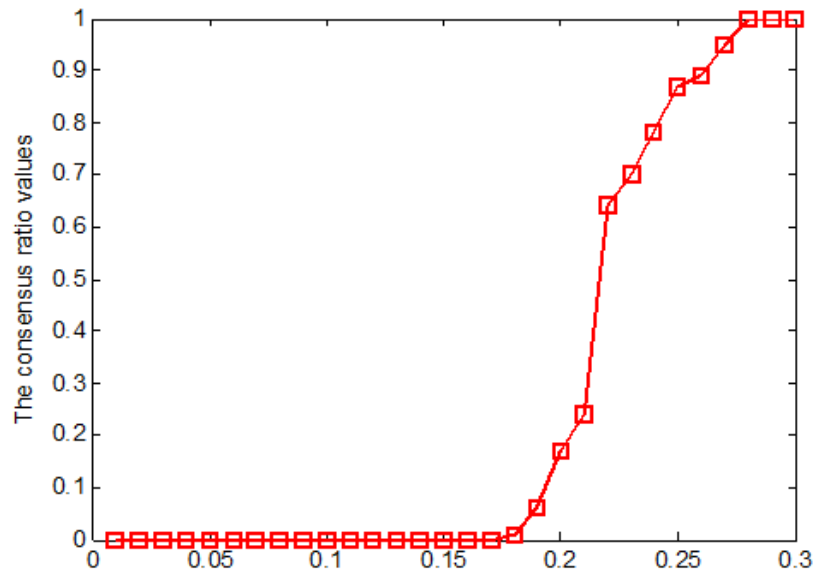

Fig. 1. The average $S R$ values for different $\alpha$ values

From Fig. 1, we obtain the following observation: the average $S R$ values increase as the $\alpha$ values increase. This observation implies that it is easier to reach a consensus as the ranges of the dynamic bounded confidences increase. Moreover, from Fig. 1, we find that a consensus will be reached when $\alpha \geq 0.3$.

Furthermore, we set different $N$ and $\alpha$ values, and run the Simulation method I 1000 times, obtaining the average $t^{*}$ values, which are shown in Fig. 2.

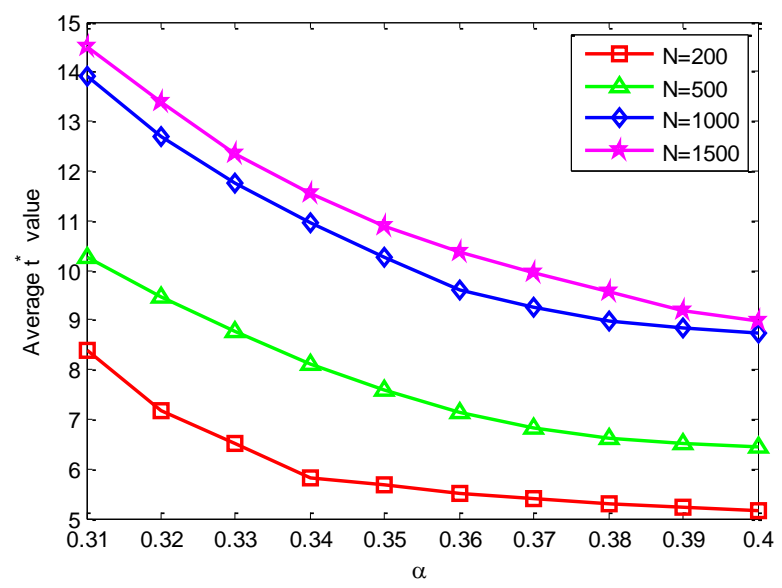

Fig. 2. The average $t^{*}$ values for different $\alpha$ values

From Fig. 2, we obtain the following observations:

(1) The average $t^{*}$ values decrease as the $\alpha$ values increase. This relation implies that the time required to a reach a consensus will decrease as the ranges of the dynamic bounded confidences increase.

(2) Less time will be required to reach a consensus for cases with smaller $N$ values.

The above observations based on Figs. 1-2 are attributed to the following reason. The 
agents have more opportunities to increase their interactions with increases in the ranges of the dynamic bounded confidences. Consequently, the opinions of the agents can reach a consensus in a shorter time.

\subsection{The effects of the widths of interval opinions}

We investigate the effects of the widths of interval opinions on the average number of fragmentations and the convergence times (i.e., the time to form the stabilized results).

Let $N F$ be the number of fragmentations, and $t^{\prime}$ be the convergence time. Let $\lambda=\max \left\{x_{i}^{U}(0)-x_{i}^{L}(0), i=1,2, \ldots, N\right\}$ be the maximum width of all the initial interval opinions. Then, we present the Simulation method II to determine the $N F$ and $t^{\prime}$ values.

\section{Simulation method II}

Input: $N, \lambda$ and $\alpha$.

Output: $N F$ and $t^{\prime}$.

Step 1: Let $t=0$, and crisp opinions $y_{i}(0) \quad(i=1,2, \ldots, N)$ are uniformly and randomly selected from $[0,1]$.

Step 2: Let $x_{i}(0)=\left[x_{i}^{L}(0), x_{i}^{U}(0)\right] \quad(i=1,2, \ldots, N)$, where $x_{i}^{L}(0)=\max \left\{0, y_{i}(0)-\delta_{i} / 2\right\}$, $x_{i}^{U}(0)=\min \left\{y_{i}(0)+\delta_{i} / 2,1\right\}$, and $\delta_{i}$ is uniformly and randomly selected from $[0, \lambda]$.

Step 3 : Dynamic bounded confidences $\varepsilon_{i}(t) \quad(i=1,2, \ldots, N)$ are uniformly and randomly selected from $[0, \alpha]$.

Step 4: Using Eqs. (7) and (8) obtains the interval opinion $x_{i}(t+1)$ at time $t+1$. If the conditions regarding stabilized results (see Definition 1) are satisfied, then let $t^{\prime}=t+1$, go to Step 5; otherwise, let $t=t+1$, then go to Step 3 .

Step 5: Output $t^{\prime}$ and $N F$.

Note 2: Step 2 guarantees that the width of interval opinion $x_{i}(0)$ is smaller than $\lambda$, and $x_{i}(0) \subseteq[0,1]$, for $i=1,2, \ldots, N$.

We set different $N, \lambda$ and $\alpha$ values, and run the Simulation method II 1000 times, obtaining the average values for $N F$ and $t^{\prime}$, which are shown in Figs. 3 and 4. 


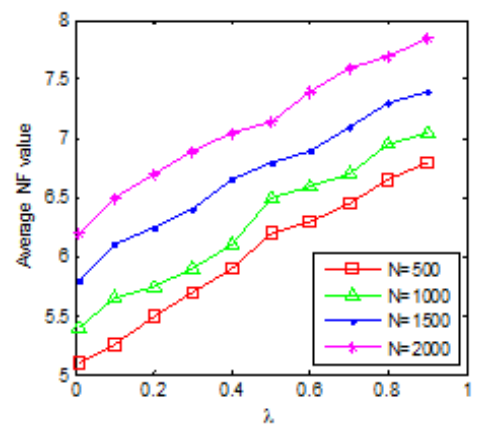

(a) $\alpha=0.1$

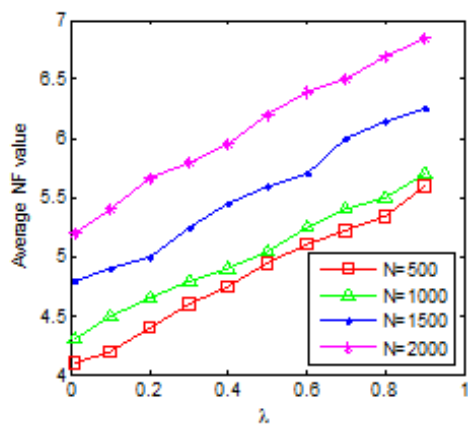

(b) $\alpha=0.15$

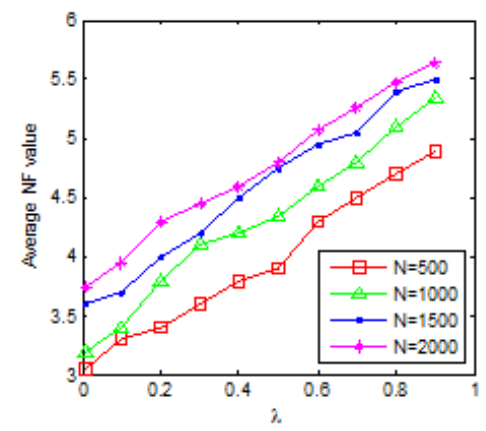

(c) $\alpha=0.2$

Fig. 3. The average $N F$ values for different $\alpha$ values

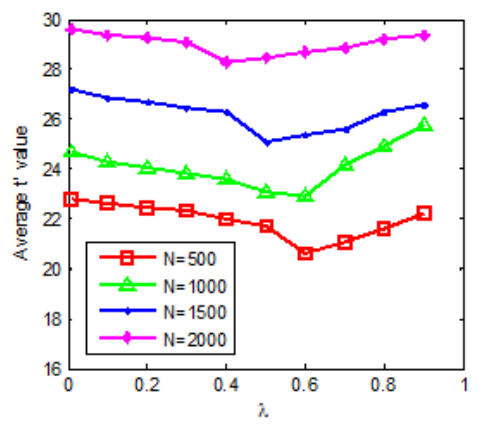

(a) $\alpha=0.1$

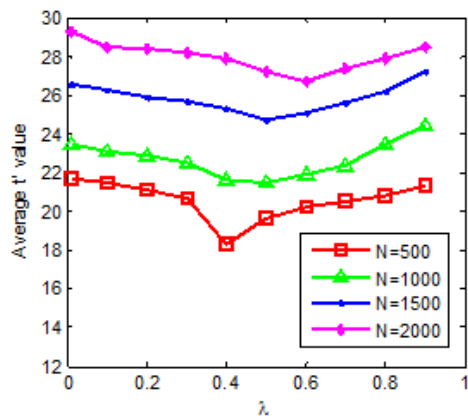

(b) $\alpha=0.15$

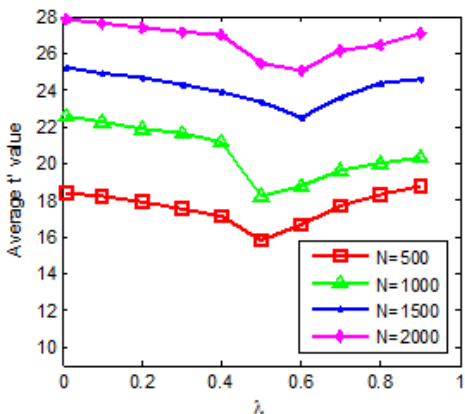

(c) $\alpha=0.2$

Fig. 4. The average $t^{\prime}$ values for different $\alpha$ values

From Figs. 3 and 4, we obtain the following three observations:

(1) Larger $\lambda$ values yield more fragmentations. This observation implies that it will be easier to form fragmentations as the widths of the interval opinions increase. This observation is related to the following reason. In the simulation, we find that with the increases of the ranges of the interval opinions, the differences among the initial interval opinions of the agents will increase. Consequently, fragmentations between the agents are formed.

(2) With increases in the $\lambda$ values, the average $t^{\prime}$ values first decrease and then increase again.

(3) More time will be required to form stable results for cases with the larger $N$ values. Moreover, larger $N$ values will result in greater numbers of fragmentations ( $N F$ ).

\subsection{Comparisons of the proposed model and the HK model}

In this subsection, we compare the proposed model with the HK model. In these comparisons, two aspects were investigated: the sequence consistency, and the conditions that result in the formation of fragmentations.

(1) Sequence consistency: 
Blondel et al. [11] proved that the HK model with homogeneous bounded confidence satisfies sequence consistency. In the following, we discuss the finding that the proposed model with dynamic bounded confidence does not satisfy sequence consistency.

Let $t^{\prime}$ be as before. If $x_{i}(0) \leq x_{j}(0)$ and $x_{i}\left(t^{\prime}\right) \leq x_{j}\left(t^{\prime}\right)$, then in this paper, we consider $A_{i}$ and $A_{j}$ to be a pair of agents that satisfy sequence consistency.

Note 3: There exist several methods for comparing two intervals [26-29]. In our study, the method presented in $[27,29]$ is used to compare two intervals. Specifically, let $x_{i}=\left[x_{i}^{L}, x_{i}^{U}\right]$ and $x_{j}=\left[x_{j}^{L}, x_{j}^{U}\right]$ be two interval numbers. The comparison of $x_{i}$ and $x_{j}$ is defined as follows: (a) $x_{i}>x_{j}$ if $P\left(x_{i}>x_{j}\right)>0.5$, and (b) $x_{i}=x_{j}$ if $P\left(x_{i}>x_{j}\right)=0.5$, where $P\left(x_{i} \geq x_{j}\right)$ is the degree of preference for $x_{i}$ over $x_{j}$, i.e.,

$$
P\left(x_{i} \geq x_{j}\right)=\frac{\max \left(0, x_{i}^{U}-x_{j}^{L}\right)-\max \left(0, x_{i}^{L}-x_{j}^{U}\right)}{x_{i}^{U}-x_{i}^{L}+x_{j}^{U}-x_{j}^{L}} .
$$

Let $P I$ be the number of pairs of agents that satisfy sequence consistency. Let $p=P I / C_{N}^{2}$; we then used the ratio $p$ to investigate whether the proposed model satisfies sequence consistency. When $p=1$, the proposed model satisfied sequence consistency. The large $p$ value indicates that the proposed model exhibits a higher ratio to satisfy sequence consistency.

Then, we present Simulation method III to calculate the ratio $p$.

\section{Simulation method III:}

Input: $N$ and $\alpha$.

Output: $p$.

Step 1: Let $t=0$ and $P I=0$. Interval opinions $x_{i}(0) \quad(i=1,2, \ldots, N)$ are uniformly and randomly selected from $[0,1]$.

Step 2: Dynamic bounded confidences $\varepsilon_{i}(t) \quad(i=1,2, \ldots, N)$ are uniformly and randomly selected from $[0, \alpha]$.

Step 3: Using Eqs. (7) and (8) obtains the interval opinion $x_{i}(t+1)$ at time $t+1$. If the conditions regarding stabilized results (see Definition 1) are satisfied, then let $t^{\prime}=t$, go to Step 4; otherwise, let $t=t+1$, go to Step 2.

Step 4: For two agents $A_{i}$ and $A_{j}(i, j=1,2, \ldots, N)$, if $x_{i}(0) \leq x_{j}(0)$ and $x_{i}\left(t^{\prime}\right) \leq x_{j}\left(t^{\prime}\right)$, then $P I=P I+1$. 
Step 5: Let $p=P I / C_{N}^{2}$, and output the $p$ value.

We set different $N$ and $\alpha$ values, and run the Simulation method III 200 times, obtaining the average $p$ values, which are shown in Fig. 5.

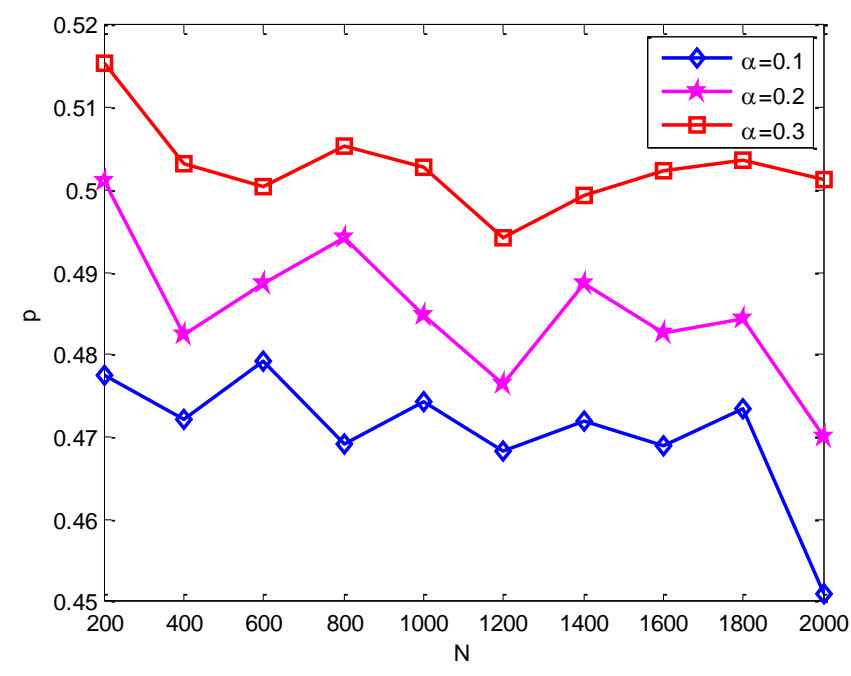

Fig. 5. The average $p$ values for different $\alpha$ values

From Fig. 5, we obtain two observations:

(i) The proposed model does not satisfy the sequence consistency.

(ii) The average $p$ values in the proposed model ranged from 0.45 to 0.52 , which implies that there exist many agents who violate sequence consistency.

The observations in Fig. 5 are attributable to two phenomena: (a) the variations in the upper and lower bounds of the interval opinions are not uniform during the process of opinion dynamics; and (b) the existence of dynamic bounded confidence causes differences in the confidence sets of the agents.

(2) Condition required to form fragmentations

In the HK model [4], the condition for the formation of fragmentations is that all of the opinions at any two neighbouring times are completely identical, and there exist at least two different opinions.

In the following, we propose a counterexample to illustrate that it is possible that the proposed model will not form fragmentations in this condition. In this example, there are 50 agents, their initial interval opinions are given by 


$$
x_{i}(0)= \begin{cases}{[0,0.2],} & i=1,2, \ldots, 10 \\ {[0.3,0.35],} & i=11,12, \ldots, 20 \\ {[0.45,0.6],} & i=21,22, \ldots, 30 . \\ {[0.7,0.75],} & i=31,32, \ldots, 40 \\ {[0.95,1],} & i=41,42, \ldots, 50\end{cases}
$$

and their initial dynamic bounded confidences are given by

$$
\varepsilon_{i}(0)= \begin{cases}0.15, & i=1,2, \ldots, 10 \\ 0.17, & i=11,12, \ldots, 20 \\ 0.2, & i=21,22, \ldots, 30 \\ 0.21, & i=31,32, \ldots, 40 \\ 0.24, & i=41,42, \ldots, 50\end{cases}
$$

Based on Eqs. (7) and (8), we find that $x_{i}(0)=x_{i}(1)$ for $i=1,2, \ldots, 50$, and there exist five interval opinions at time $t=1$.

Let $\alpha=0.25$. Based on the proposed model, we obtain the evolutions of the interval opinions, which are shown in Fig. 6. The red and blue lines in Fig. 6 represent the evolutions of the lower and upper bounds of interval opinions, respectively.

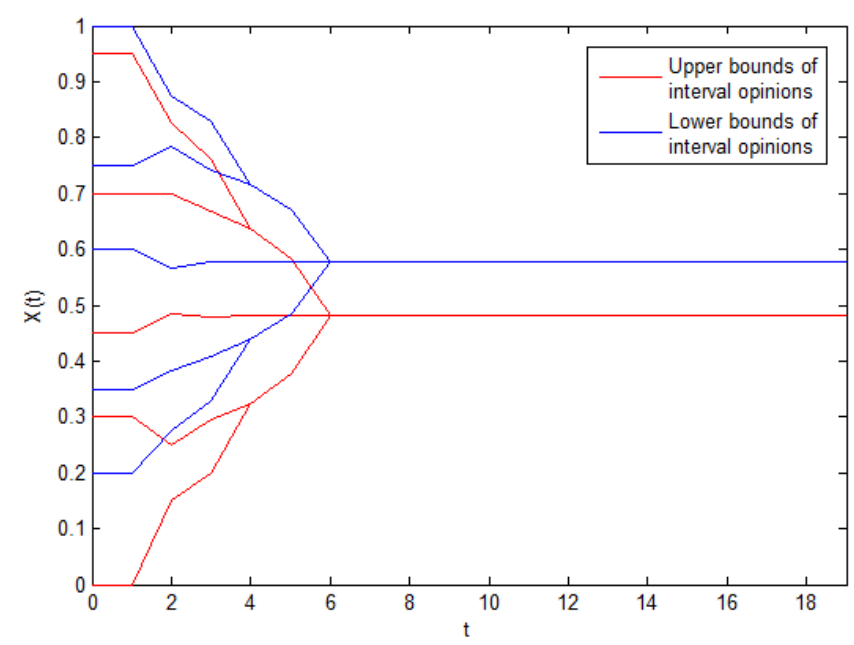

Fig. 6. The evolutions of the interval opinions in the proposed model

From Fig. 6, we obtain the following observation: the evolutions of the interval opinions continue after the time $t=1$, and a consensus is finally reached in opinion dynamics. This implies that the proposed model does not satisfy the conditions for forming fragmentations in the HK model.

The observation in Fig. 6 can be explained by the dynamic bounded confidence. Changes in dynamic bounded confidences often cause changes of opinions. Consequently, the conditions for the formation of fragmentations of the HK model and the proposed model are not identical. 


\section{Conclusions}

In the present study, we propose a novel model, termed the interval opinion dynamics with the dynamic bounded confidence. The primary contributions of this study are as follows:

(1) In contrast to the original $\mathrm{HK}$ model, in the proposed model, the agents express interval opinions rather than crisp number opinions and employ dynamic bounded confidences in specified intervals.

(2) We propose some conditions that are sufficient for the formation of consensuses or fragmentations in the proposed opinion dynamics.

(3) Through the simulation experiments, we investigate the effects of dynamic bounded confidence on opinion dynamics and the effects of the widths of the interval opinions on opinion dynamics, and also compared our proposal with the original HK model.

In our view, the opinion dynamics models are related to the consensus-reaching processes of group decision-making problems [30-41]. In particular, with the rapid development of information technology and social economic, proposals related to and studies of large-scale group decision-making have increasing been performed [42]. We argue that opinion dynamics models will potentially be tool that support consensus-reaching in large-scale group decision-making problems. This issue is now under investigation.

\section{Acknowledgments}

This work was supported in part by the NSF of China via grant 71171160 and in part by Sichuan University via grant skqx201308.

\section{References}

[1] M. Afshar, M. Asadpour, Opinion formation by informed agents. Journal of Artificial Societies and Social Simulation 13(4) (2010).

[2] P.L. Krapivsky, S. Redner, Dynamics of majority rule in two-State interacting spin systems. Physical Review Letters 90(23) (2003) 1-4.

[3] M. Lewenstein, A. Nowak, B. Latané, Statistical mechanics of social impact. Physical Review A 45(2) (1992) 763-776.

[4] R. Hegselmann, U. Krause, Opinion dynamics and bounded confidence models, analysis, and simulation. Journal of Artificial Societies and Social Simulation 5(3) (2002).

[5] G. Deffuant, D. Neau, F. Amblard, G. Weisbuch, Mixing beliefs among interacting agents. Advances in Complex Systems 3 (2000) 87-98.

[6] R. Axelrod, The dissemination of culture: A model with local convergence and global polarization. Journal of Conflict Resolution, 41 (2) (1997) 203-226. 
[7] D. Lim, H. Lee, H. Zo, A. Ciganek, Opinion formation in the digital divide. Journal of Artificial Societies and Social Simulation, 17(1) (2014).

[8] G. Weisbuch, Bounded confidence and social networks. The European Physical Journal BCondensed Matter and Complex Systems 38(2) (2004) 339-343.

[9] J. Lorenz, Continuous opinion dynamics under bounded confidence: A survey. International Journal of Modern Physics C 18(12) (2007) 1819-1838.

[10]D. Urbig, J. Lorenz, H. Herzberg, Opinion dynamics: The effect of the number of peers met at once. Journal of Artificial Societies and Social Simulation 11(2) (2008).

[11]V.D. Blondel, J. M. Hendrickx, J. N. Tsitsiklis, On Krause's multi-agent consensus model with state-dependent connectivity. IEEE Transactions on Automatic Control 54(11) (2009) 2586-2597.

[12]I.C. Morarescu, A. Girard, Opinion dynamics with decaying confidence: Application to community detection in graphs. IEEE Transactions on Automatic Control 56(8) (2011) 1862-1873.

[13]G. Fu, W. Zhang, Z. Li, Opinion dynamics of modified Hegselmann-Krause model in a group-based population with heterogeneous bounded confidence. Physica A: Statistical Mechanics and its Applications 419 (2015): 558-565..

[14]J. Lorenz, Heterogeneous bounds of confidence: Meet, discuss and find consensus!. Complexity 15(4) (2010) 43-52.

[15]F. Ceragioli, P. Frasca, Continuous and discontinuous opinion dynamics with bounded confidence. Nonlinear Analysis: Real World Applications 13(3) (2012) 1239-1251.

[16]A. Mirtabatabaei, F. Bullo, On opinion dynamics in heterogeneous networks. IEEE on American Control Conference, (2011) 2807-2812.

[17]S. Fortunato, V. Latora, A. Pluchino, A. Rapisarda, Vector opinion dynamics in a bounded confidence consensus model. International Journal of Modern Physics C 16(10) (2005) $1535-1551$.

[18]J. Lorenz, Consensus strikes back in the Hegselmann-Krause model of continuous opinion dynamics under bounded confidence. Journal of Artificial Societies and Social Simulation (2006) 9(1).

[19]J. Lorenz, A stabilization theorem for dynamics of continuous opinions. Physica A: Statistical Mechanics and its Applications 355(1) (2005) 217-223.

[20]S. Kurz, J. Rambau, On the Hegselmann-Krause conjecture in opinion dynamics. Journal of Difference Equations and Applications 17(6) (2011) 859-876.

[21]Y. Shang, An agent based model for opinion dynamics with random confidence threshold. 
Communications in Nonlinear Science and Numerical Simulation 19(10) (2014) 3766-3777.

[22]Y.M. Wang, J.B. Yang, D.L. Xu, Interval weight generation approaches based on consistency test and interval comparison matrices. Applied Mathematics and Computation 167(1) (2005) 252-273.

[23]T.L. Saaty, L.G. Vargas, Uncertainty and rank order in the analytic hierarchy process. European Journal of Operational Research 32(1) (1987) 107-117.

[24]Y.C. Dong, G.Q. Zhang, W.C. Hong, S. Yu, Linguistic computational model based on 2-tuples and intervals. IEEE Transactions on Fuzzy Systems, 21(6) (2013) 1006-1018.

[25]J.C. Dittmer, Consensus formation under bounded confidence. Nonlinear Analysis: Theory, Methods \& Applications, 47(7) (2001) 4615-4621.

[26]A. Senguta, T.K. Pal, On comparing interval numbers. European Journal of Operation Research 127(1) (2000) 28-43.

[27]Y.M. Wang, J.B. Yang, D.L. Xu, A preference aggregation method through the estimation of utility intervals. Computers \& Operations Research 32(8) (2005) 2027-2049.

[28]P. Sevastianov, Numerical methods for interval and fuzzy number comparison based on the probabilistic approach and Dempster-Shafer theory. Information Sciences 177(21) (2007) 4645-4661.

[29] R.M. Rodríguez, L. Martínez, F. Herrera, Hesitant fuzzy linguistic term set for decision making, IEEE Transactions on Fuzzy Systems. 20 (1) (2012) 109-119.

[30]F. Herrera, E. Herrera-Viedma, J.L. Verdegay, A model of consensus in group decision making under linguistic assessments. Fuzzy Sets and Systems 1(78) (1996) 73-87.

[31]F. Herrera, E. Herrera-Viedma, J.L. Verdegay, A rational consensus model in group decision making using linguistic assessments. Fuzzy Sets and Systems 1(88) (1997) $31-49$.

[32]E. Herrera-Viedma, F.J. Cabrerizo, J. Kacprzyk, W. Pedrycz, A review of soft consensus models in a fuzzy environment. Information Fusion, 17 (2014) 4-13.

[33]I. Palomares, F.J. Estrella, L. Martinez, F. Herrera, Consensus under a fuzzy context: Taxonomy, analysis framework AFRYCA and experimental case of study. Information Fusion, 20 (2014) 252-271.

[34]I. Palomares, L. Martínez, A semisupervised multi-agent system model to support consensus reaching processes, IEEE Transactions on Fuzzy Systems 4(22) (2014) 762-777.

[35]E. Herrera-Viedma, J.L. García-Lapresta, J. Kacprzyk, M. Fedrizzi, H. Nurmi, S. 
Zadrozny (Eds.), Consensual Processes, STUDFUZZ, vol. 267, Springer-Verlag, Berlin, 2011.

[36]F. Chiclana, J.M.Tapia García, M.J. del Moral, E. Herrera-Videdma, A statistical comparative study of different similarity measures of consensus in group decision making, Information Sciences 221 (2013) 110-123.

[37]Y.C. Dong, X. Chen, F. Herrera, Minimizing adjusted simple terms in the consensus reaching process with hesitant linguistic assessments in group decision making, Information Sciences 297 (2015) 95-117.

[38]G.Q. Zhang, Y.C. Dong, Y.F. Xu, Consistency and consensus measures for linguistic preference relation based on distribution assessments, Information Fusion 17 (2014) $46-55$.

[39]Y.C. Dong, Y.F. Xu, H.Y. Li, B. Feng, The OWA-based consensus operator under linguistic representation models using position indexes, European Journal of Operational Research 203(2) (2010) 455-463.

[40]X. Chen, H.J. Zhang, Y.C. Dong, The fusion process with heterogeneous preference structures in group decision making: A survey, Information Fusion 24 (2015) 72-83.

[41] S.W. Chen, J. Liu, H. Wang, J.C. Augusto, Ordering based decision making-A survey, Information Fusion 14(4) (2013) 521-531.

[42]I. Palomares, L. Martínez, F. Herrera, A consensus model to detect and manage noncooperative behaviors in large-scale group decision making, IEEE Transactions on Fuzzy Systems 22 (3) (2014) 516-530. 


\section{Appendix}

\section{A1. Proof of Lemma 1.}

Proof. According to Eqs. (5) and (6), $x_{i}(t+1) \in \operatorname{conv}\left\{x_{1}(t), \ldots, x_{N}(t)\right\}, \quad i=1,2, \ldots, N$.

Thus, the distance $d\left(x_{i}(t+1), x_{j}(t+1)\right)$ is given as follows:

$$
\begin{aligned}
& d\left(x_{i}(t+1), x_{j}(t+1)\right)=\left[\frac{1}{2}\left[\left(\sum_{k=1}^{N}\left(w_{i k}(t)-w_{j k}(t)\right) x_{k}^{L}(t)\right)^{2}+\left(\sum_{k=1}^{N}\left(w_{i k}(t)-w_{j k}(t)\right) x_{k}^{U}(t)\right)^{2}\right]\right]^{1 / 2} \\
& =\left[\frac { 1 } { 2 } \left[\left(\sum_{k=1}^{N}\left(w_{i k}(t)-\min \left\{w_{i k}(t), w_{j k}(t)\right\}\right) x_{k}^{L}(t)-\left(w_{j k}(t)-\min \left\{w_{i k}(t), w_{j k}(t)\right\}\right) x_{k}^{L}(t)\right)^{2}+\right.\right. \\
& \left.\quad\left(\sum_{k=1}^{N}\left(w_{i k}(t)-\min \left\{w_{i k}(t), w_{j k}(t)\right\}\right) x_{k}^{U}(t)-\left(w_{j k}(t)-\min \left\{w_{i k}(t), w_{j k}(t)\right\}\right) x_{k}^{U}(t)\right)^{2}\right]^{1 / 2} \\
& \leq\left[\frac{1}{2}\left[\left(1-\sum_{k=1}^{N} \min \left\{w_{i k}(t), w_{j k}(t)\right\}\right)^{2}\left(\underline{x}^{L}(t)-\bar{x}^{L}(t)\right)^{2}+\left(1-\sum_{k=1}^{N} \min \left\{w_{i k}(t), w_{j k}(t)\right\}\right)^{2}\left(\underline{x}^{U}(t)-\bar{x}^{U}(t)\right)^{2}\right]^{1 / 2}\right. \\
& =\left[\left(1-\sum_{k=1}^{N} \min \left\{w_{i k}(t), w_{j k}(t)\right\}\right)^{2} \frac{1}{2}\left[\left(\underline{x}^{L}(t)-\bar{x}^{L}(t)\right)^{2}+\left(\underline{x}^{U}(t)-\bar{x}^{U}(t)\right)^{2}\right]^{1 / 2}\right. \\
& =\left(1-\min _{1 \leq i, j \leq N} \sum_{k=1}^{N}\left\{w_{i k}(t), w_{j k}(t)\right\}\right) \sqrt{\frac{\left(\underline{x}^{L}(t)-\bar{x}^{L}(t)\right)^{2}+\left(\underline{x}^{U}(t)-\bar{x}^{U}(t)\right)^{2}}{2}} \\
& =\left(1-\min _{1 \leq i, j \leq N} \sum_{k=1}^{N}\left\{w_{i k}(t), w_{j k}(t)\right\}\right) d\left(\underline{x}^{2}(t), \bar{x}(t)\right)
\end{aligned}
$$

Due to the arbitrariness of $x_{i}(t+1)$ and $x_{j}(t+1)$, we can further obtain

$$
F\left(\operatorname{conv}\left\{x_{1}(t), \ldots, x_{N}(t)\right\}\right) \leq\left(1-\min _{1 \leq i, j \leq N} \sum_{k=1}^{N}\left\{w_{i k}(t), w_{j k}(t)\right\}\right) d(\underline{x}(t), \bar{x}(t)) .
$$

This completes the proof of Lemma $\mathbf{1}$.

\section{A2. Proof of Theorem 1.}

Proof. (1) Based on Lemma 1, we obtain

$$
F\left(\operatorname{conv}\left\{x_{1}(t), \ldots, x_{N}(t)\right\}\right) \leq\left(1-\min _{1 \leq i, j \leq N} \sum_{k=1}^{N}\left\{b_{i k}(t-s), b_{j k}(t-s)\right\}\right) d(\underline{x}(s), \bar{x}(s)), t>s .
$$

Because $\sum_{k=1}^{N} \min \left\{b_{i k}\left(t_{m}, t_{m-1}\right), b_{j k}\left(t_{m}, t_{m-1}\right)\right\} \geq \delta_{m}$, for time $t>t_{m+1}$, we have

$$
F\left(\operatorname{conv}\left\{x_{1}(t), \ldots, x_{N}(t)\right\}\right) \leq\left(1-\delta_{m}\right)\left(1-\delta_{m-1}\right) \cdots\left(1-\delta_{0}\right) d(\underline{x}(0), \bar{x}(0))
$$

Because $1-\delta_{m} \leq e^{-\delta_{m}}$ for $\delta_{m} \geq 0$. Hence,

$$
F\left(\operatorname{conv}\left\{x_{1}(t), \ldots, x_{N}(t)\right\}\right) \leq e^{-\delta_{m}} e^{-\delta_{m-1}} \cdots e^{-\delta_{0}} d(\underline{x}(0), \bar{x}(0))=e^{-\sum_{r=1}^{m} \delta_{r}} d(\underline{x}(0), \bar{x}(0))
$$


Because $\lim _{r \rightarrow \infty} \sum_{m=1}^{r} \delta_{m}=\infty, \quad F\left(\operatorname{conv}\left\{x_{1}(t), \ldots, x_{N}(t)\right\}\right) \leq 0$.

We further obtain $F\left(\operatorname{conv}\left\{x_{1}(t), \ldots, x_{N}(t)\right\}\right)=0$. According to the meaning of $F\left(\operatorname{conv}\left\{x_{1}(t), \ldots x_{N}(t)\right\}\right)$, a consensus between the agents is reached, i.e., $\lim _{t \rightarrow \infty} x_{i}(t)=x^{*}$ for $i=1,2, \ldots, N$.

(2) Because confidence chains exist from $A_{i}$ to $A_{k}$ and $A_{J}$ to $A_{k}$ between the times $(m-1) h$ and $m h$, we can obtain

$$
I_{i}\left(m h, x_{i}(m h)\right) \cap I_{j}\left(m h, x_{j}(m h)\right)=\varnothing \text {, e.g. } A_{k} \in I_{i}\left(m h, x_{i}(m h)\right) \cap I_{j}\left(m h, x_{j}(m h)\right) .
$$

Furthermore, we have $\sum_{k=1}^{N} \min \left\{b_{i k}(m h,(m-1) h), b_{j k}(m h,(m-1) h)\right\} \geq\left(\frac{1}{N}\right)^{h}$. Obviously, $\left(\frac{1}{N}\right)^{h}>0$, and when $m \rightarrow \infty, m\left(\frac{1}{N}\right)^{h} \rightarrow \infty$.

According to Lemma 1 and Theorem 1, we can obtain $\lim _{t \rightarrow \infty} d\left(x_{i}(t), x_{j}(t)\right)=0$, which indicates that any pair of agents hold the same opinion; thus, a consensus is reached.

(3) When a consensus is not reached at time $t$, then there exists one agent whose confidence set does not include all of the agents.

Without a loss of generality, we assume $\left|I\left(i, x_{i}(t)\right)\right|<N-1, d\left(x_{i}(t), x_{k}(t)\right)>\varepsilon_{i}(t)$, and $\max _{j=1,2, \ldots, N} d\left(x_{i}(t), x_{j}(t)\right) \geq d\left(x_{i}(t), x_{k}(t)\right)$.

If $\Delta \varepsilon_{i}(t) \geq \max _{j=1,2, \ldots, N} d\left(x_{i}(t), x_{j}(t)\right)-\varepsilon_{i}(t)$, it implies that

$$
\varepsilon_{i}(t+1)=\varepsilon_{i}(t)+\Delta \varepsilon_{i}(t) \geq \max _{j=1,2, \ldots, N} d\left(x_{i}(t), x_{j}(t)\right) .
$$

Then $\left|I\left(i, x_{i}(t+1)\right)\right|=N$ is satisfied at time $t+1$. Clearly, a consensus is reached at time $t+1$.

This completes the proof of Theorem 1. $\square$

\section{A3. Proof of Lemma 2.}

Proof. Let $n^{*}=t_{0}-t_{1}$ because $\mu\left(B\left(t_{0}, t_{1}\right)\right)=\mu\left(A\left(t_{1}-1\right) A\left(t_{1}-2\right) \cdots A\left(t_{0}\right)\right)$; thus, we obtain

$$
\mu\left(B\left(t_{0}, t_{1}\right)\right)=\mu\left(A\left(t_{1}-1\right) A\left(t_{1}-2\right) \cdots A\left(t_{0}\right)\right) \geq \mu\left(A\left(t_{1}-1\right)\right) \mu\left(A\left(t_{1}-2\right)\right) \cdots \mu\left(A\left(t_{0}\right)\right) \geq(1 / N)^{n^{*}} .
$$

If $n^{*} \leq N^{2}-N+2$, then $\mu\left(B\left(t_{0}, t_{1}\right)\right) \geq(1 / N)^{N^{2}-N+2}$ naturally holds.

Because $\varepsilon_{1}(t)=\varepsilon_{2}(t)=\cdots=\varepsilon_{N}(t)$, then the weight matrix $W(t)$ at time $t$ satisfies the following two conditions: (1) if $w_{i j}(t)=0$, then $w_{j i}(t)=0$; and (2) if $w_{i j}(t)>0$, then 
$w_{j i}(t)<0$.

For any two matrices $B$ and $C, B, C \in \square_{\geq 0}^{n \times n}$, proof of the following conclusion is needed:

If $\mu(C B)<\mu(B)$, then $\exists i, j,(c b)_{i j}>0$ and $b_{i j}=0$.

To prove this conclusion, we assume that the zero patterns of $B C$ and $B$ are the same and that $\mu(C B) \geq \mu(B)$.

According to this assumption, if $b_{k j}=0$, then $(c b)_{k j}=0$, and $(c b)_{k j}$ is rewritten as:

$$
(c b)_{k j}=\sum_{l=1}^{n} c_{k d} b_{l j}=0 \stackrel{b_{i j}>0}{\longrightarrow} c_{k i}=0 \Rightarrow c_{i k}=0
$$

Let $N^{\prime}=\left\{i \mid(c b)_{i j}>0\right.$ and $\left.c_{i j}>0\right\}$ be the set of indices. Obviously, we have $\sum_{k \in N^{\prime}, c_{k j}>0} b_{i k}=1$. Thus,

$$
(c b)_{i j}=\sum_{k \in N^{\prime}, b_{k j}>0} c_{i k} b_{k j} \geq\left(\min _{k \in N^{\prime}, b_{k j}>0} b_{k j}\right)\left(\sum_{k \in N^{\prime}, b_{k j}>0} c_{i k}\right) \geq \min _{k \in N^{\prime}, b_{k j}>0} b_{k j} \geq \mu(B) .
$$

Therefore, after one multiplication between the two accumulated weight matrices, at least one zero entry will lose. Because the diagonal elements of the accumulated weight matrix at any time are always nonzero, the number of zero elements in the accumulated weight matrix is equal to at most $N^{2}-N+1$. Therefore, $\mu\left(B\left(t_{0}, t_{1}\right)\right) \geq(1 / N)^{N^{2}-N+2}$.

This completes the proof of Lemma 2.

\section{A4. Proof of Lemma 2.}

Proof. Based on Eqs. (9) and (10), the interval opinions $x_{i}=\left[x_{i}^{L}(t+1), x_{i}^{U}(t+1)\right]$ can written as

$$
x_{i}^{L}(t+1)=\sum_{j=1}^{N} b_{i j}(t, 0) x_{j}^{L}(0), \quad i=1,2, \ldots, N
$$

and

$$
x_{i}^{U}(t+1)=\sum_{j=1}^{N} b_{i j}(t, 0) x_{j}^{U}(0), \quad i=1,2, \ldots, N
$$

where $b_{i j}(t, 0)>0$ and $\sum_{j=1}^{N} b_{i j}(t, 0)=1$.

Because a consensus is reached at time $t+1$,

$$
x^{c}=\left[x^{c L}, x^{c U}\right]=\left[x_{i}^{L}(t+1), x_{i}^{U}(t+1)\right], \quad i=1,2, \ldots, N .
$$

Moreover, the width $L\left(x^{c}\right)$ can be written as 


$$
\begin{aligned}
& L\left(x^{c}\right)=x_{i}^{U}(t+1)-x_{i}^{L}(t+1)=\sum_{j=1}^{N} b_{i j}(t, 0)\left(x_{j}^{U}(0)-x_{j}^{L}(0)\right) \\
& \leq \max _{j=1,2, \ldots, N}\left\{b_{i j}(t, 0)\right\} L\left(x_{\max }^{(0)}\right)+\left(1-\max _{j=1,2, \ldots, N}\left\{b_{i j}(t, 0)\right\}\right) L\left(x_{\min }^{(0)}\right)
\end{aligned}
$$

(1) If $t<N^{2}-N+2$, the according to Lemma 2, we have $\min _{j=1,2, \ldots, N}\left\{b_{i j}(t, 0)\right\} \geq(1 / N)^{t}$. We can then determine the maximum accumulated weight as

$$
\max _{j=1,2, \ldots, N}\left\{b_{i j}(t, 0)\right\} \leq 1-(N-1) \min _{j=1,2, \ldots, N}\left\{b_{i j}(t, 0)\right\}=1-(N-1)(1 / N)^{t}
$$

Furthermore, we obtain

$$
L\left(x^{c}\right) \leq\left[1-(N-1)(1 / N)^{t}\right] L\left(x_{\max }^{(0)}\right)+(N-1)(1 / N)^{t} L\left(x_{\min }^{(0)}\right)
$$

(2) If $t \geq N^{2}-N+2$, and $\varepsilon_{1}(t)=\varepsilon_{2}(t)=\cdots=\varepsilon_{N}(t)$, then according to Lemma 2, we have $\min _{j=1,2, \ldots, N}\left\{b_{i j}(t, 0)\right\}=(1 / N)^{N^{2}-N+2}$.

Because $b_{i j}(t, 0)>0$, we can then determine the maximum accumulated weight as

$$
\max _{j=1,2, \ldots, N}\left\{b_{i j}(t, 0)\right\}=1-(N-1) \min _{j=1,2, \ldots, N}\left\{b_{i j}(t, 0)\right\}=1-(N-1)(1 / N)^{N^{2}-N+2} .
$$

Furthermore, we obtain

$$
L\left(x^{c}\right) \leq\left[1-(N-1)(1 / N)^{N^{2}-N+2}\right] L\left(x_{\max }^{(0)}\right)+(N-1)(1 / N)^{N^{2}-N+2} L\left(x_{\min }^{(0)}\right) .
$$

(3) We can adopt a proof similar to (1).

This completes the proof of Theorem 2.

\section{A5. Proof of Theorem 3.}

Proof. Let $c_{g}(t)$ be the subset of agents who hold the interval opinion $\bar{x}^{g}(t)$ at time $t$, $c_{g}(t) \subseteq A, \bigcup_{g=1}^{f(t)} c_{g}(t)=A, \quad c_{g}(t) \cap c_{h}(t)=\varnothing, \quad g, h=1,2, \ldots, f(t)$.

(1) Let $A_{k} \in c_{g}(t)$ and $A_{u} \in c_{h}(t)$; thus, we can easily see that $d\left(\bar{x}^{g}(t), \bar{x}^{h}(t)\right)>\max$ $\left\{\varepsilon_{k}(t), \varepsilon_{u}(t)\right\}$ always holds. Because $\varepsilon_{k}(\tilde{t}) \leq \varepsilon_{k}(t)$ and $\varepsilon_{u}(\tilde{t}) \leq \varepsilon_{u}(t), \quad d\left(\bar{x}^{g}(t), \bar{x}^{h}(t)\right)>$ $\varepsilon_{i}(\tilde{t})$. Thus, the agents in the sets $c_{g}(t)$ and $c_{h}(t)$ do no merge at time $\tilde{t}, \tilde{t} \geq t$.

Let $A_{l} \in c_{g}(t)$. We obtain $d\left(x_{k}(t), x_{l}(t)\right)=0$. Although $\varepsilon_{k}(\tilde{t}) \leq \varepsilon_{k}(t)$ and $\varepsilon_{l}\left(t^{\prime}\right) \leq$ $\varepsilon_{l}(t)$, the agents $A_{i}$ and $A_{j}$ still mutually affect each other. Furthermore, the agents in the set $c_{g}(t)$ still hold the same opinions at time $\tilde{t}$. Thus, we further obtain $\bar{x}^{g}(t)=\bar{x}^{g}(\tilde{t}), g=1,2, \ldots, f(t)$. All of the interval opinions at time $t$ are stabilized, and fragmentations are formed. 
(2) For any two subsets $c_{g}(t)$ and $c_{h}(t)$, if $d\left(\bar{x}^{g}(t), \bar{x}^{h}(t)\right)>\alpha$, then it is implied that every agent in the set $c_{g}(t)\left(c_{h}(t)\right)$ has no neighbours in the other subsets; thus, $\bar{x}^{g}(t)=\bar{x}^{g}(t+1) \quad\left(\bar{x}^{h}(t)=\bar{x}^{h}(t+1)\right)$ for $t=1,2, \ldots$. Clearly, all of the interval opinions at time $t$ are stabilized, and fragmentations are formed.

This completes the proof of Theorem 3 . $\square$ 